\title{
Gamma-Band Synchronization in the Macaque Hippocampus and Memory Formation
}

\author{
Michael J. Jutras, ${ }^{1,2}$ Pascal Fries, ${ }^{4,5}$ and Elizabeth A. Buffalo ${ }^{1,2,3}$ \\ ${ }^{1}$ Yerkes National Primate Research Center, ${ }^{2}$ Neuroscience Program, Emory University, and ${ }^{3}$ Department of Neurology, Emory University School of \\ Medicine, Atlanta, Georgia 30329, ${ }^{4}$ Donders Institute for Brain, Cognition and Behaviour, Radboud University Nijmegen, 6525 EN Nijmegen, The \\ Netherlands, and ${ }^{5}$ Ernst Strüngmann Institute in Cooperation with Max Planck Society, 60528 Frankfurt, Germany
}

Increasing evidence suggests that neuronal synchronization in the gamma band $(30-100 \mathrm{~Hz})$ may play an important role in mediating cognitive processes. Gamma-band synchronization provides for the optimal temporal relationship between two signals to produce the long-term synaptic changes that have been theorized to underlie memory formation. Although neuronal populations in the hippocampus oscillate in the gamma range, the role of these oscillations in memory formation is still unclear. To address this issue, we recorded neuronal activity in the hippocampus while macaque monkeys performed a visual recognition memory task. During the encoding phase of this task, hippocampal neurons displayed gamma-band synchronization. Additionally, enhanced gamma-band synchronization during encoding predicted greater subsequent recognition memory performance. These changes in synchronization reflect enhanced coordination among hippocampal neurons and may facilitate synaptic changes necessary for successful memory encoding.

\section{Introduction}

Accumulating evidence suggests that, along with changes in the firing rates of individual neurons, the precise timing of neuronal activity may play an important role in cognition. Synchronization of neuronal activity in the gamma frequency band (30-100 $\mathrm{Hz}$ ) has been related to selective attention (Fries et al., 2001, 2008; Bichot et al., 2005; Taylor et al., 2005; Womelsdorf et al., 2006; Buschman and Miller, 2007) and working memory (Pesaran et al., 2002). Additionally, studies of intracranial electroencephalography in epileptic patients suggest that gamma-band synchronization may be an important component in successful memory encoding (Fell et al., 2001; Sederberg et al., 2007a). By aligning periods of inhibition, gamma-band synchronization establishes precise coordination in the spike times of neurons responding to behaviorally relevant stimuli (Mishra et al., 2006; Womelsdorf et al., 2007). Gamma-band synchronization among a group of neurons ensures that presynaptic spikes arrive at mutual downstream targets within $\sim 10 \mathrm{~ms}$ of each other. Since mutual synaptic input is followed reliably by postsynaptic spikes, this precise temporal relationship provides the necessary conditions for long-term changes in synaptic strength, which is considered to be one of the primary information storage principles in the brain (Markram et al., 1997; Bi and Poo, 1998). However, to date, there has been

Received Feb. 6, 2009; revised Aug. 31, 2009; accepted Sept. 2, 2009.

This work was supported by the Yerkes National Primate Research Center through National Institutes of Health Base Grant RR00165, Ernst Strüngmann Institute, Emory Alzheimer's Disease Research Center Grant AG025688 (E.A.B.), the National Institute of General Medical Sciences (M.J.J.), and National Institute of Mental Health Grants MH080007 (E.A.B.) and MH082559 (M.J.J.). We thank E. Stanley and M. Tompkins for technical assistance, and R.D. Burwell, R. Desimone, J. R. Manns, and L. R. Squire for comments on this manuscript.

Correspondence should be addressed to Elizabeth A. Buffalo, Yerkes National Primate Research Center, 954 Gatewood Road, Atlanta, GA 30329. E-mail: elizabeth.buffalo@emory.edu.

DOI:10.1523/JNEUROSCI.0640-09.2009

Copyright $\odot 2009$ Society for Neuroscience 0270-6474/09/2912521-11\$15.00/0 little direct evidence for a relationship between gamma-band synchronization among hippocampal neurons and memory formation.

Recognition memory, the ability to perceive a recently encountered item as familiar, is degraded after damage to the hippocampus in humans and monkeys (Zola et al., 2000; Manns et al., 2003), although findings regarding the role of the hippocampus in recognition memory have not always been consistent across laboratories (Murray and Mishkin, 1998; Baxter and Murray, 2001a,b; Zola and Squire, 2001; Nemanic et al., 2004). To add to this controversy, only a very small number of neurons have been reported to display recognition memory signals in the hippocampus proper (Brown et al., 1987; Rolls et al., 1989, 1993; Riches et al., 1991; Xiang and Brown, 1998) (but see Rutishauser et al., 2006). The apparent inconsistency between the findings from lesion and physiology studies raises doubt about the contribution of the hippocampus to recognition memory.

All of these previous neurophysiological studies examined changes in firing rate that might act as a signal for recognition memory. However, it is possible that recognition signals in the hippocampus may take the form of enhanced neuronal synchronization among groups of neurons. Here, we examined the relationship between neuronal synchronization among hippocampal neurons and recognition memory performance on the visual preferential looking task (VPLT) in monkeys. This task has been shown to depend on the integrity of the hippocampus in both monkeys (Pascalis and Bachevalier, 1999; Zola et al., 2000; Nemanic et al., 2004) and humans (McKee and Squire, 1993). We report that hippocampal neurons show gamma-band synchronization during encoding that is positively correlated with subsequent recognition memory performance. These changes in synchronization reflect enhanced interaction among hippocampal neurons and 
may provide a mechanism for the synaptic changes necessary for successful memory formation.

\section{Materials and Methods}

Procedures were performed in accordance with National Institutes of Health guidelines and were approved by the Emory University Institutional Animal Care and Use Committee. Neuronal recordings were made in two adult male rhesus monkeys (Macaca mulatta), obtained from the breeding colony at the Yerkes National Primate Research Center. Their mean weight at the start of the experiment was $6.8 \pm 1.1 \mathrm{~kg}$, and their mean age was 4 years and 5 months. Before implantation of recording hardware, monkeys were scanned with magnetic resonance imaging (MRI) to localize the hippocampus and to guide placement of the recording chamber. Using this information, a cilux plastic chamber (Crist Instrument) for recording neural activity, and a titanium post for holding the head were surgically implanted. Postsurgical MRI was performed to localize recording sites.

\section{Behavioral testing procedures}

During testing, each monkey sat in a dimly illuminated room, $60 \mathrm{~cm}$ from a 19 inch cathode ray tube monitor that had a resolution of $800 \times$ 600 pixels and a screen refresh rate of $120 \mathrm{~Hz}$ non-interlaced. Eye movements were recorded using a noninvasive infrared eye-tracking system (ISCAN).

Stimuli were presented using experimental control software (CORTEX; www.cortex.salk.edu). At the beginning of each recording session, the monkey performed an eye position calibration task, which involved holding a touch-sensitive bar while fixating a small $\left(0.3^{\circ}\right)$ gray fixation point, presented on a dark background at various locations on the monitor. The monkey was required to maintain fixation within a $3^{\circ}$ window until the fixation point changed to an equiluminant yellow at a randomly chosen time between 500 and $1100 \mathrm{~ms}$ after fixation onset. The monkey was required to release the touch-sensitive bar within $500 \mathrm{~ms}$ of the color change for delivery of a drop of applesauce. During this task, the gain and offset of the oculomotor signals were adjusted so that the computed eye position matched targets that were a known distance from the central fixation point.

\section{Visual preferential looking task}

After the calibration task, the monkey was tested on the VPLT (see Fig. $1 A)$. The monkey initiated each trial by fixating a white cross $\left(1^{\circ}\right)$ at the center of the computer screen. After maintaining fixation on the cross for $1 \mathrm{~s}$, the cross disappeared and the picture stimulus $\left(11^{\circ}\right)$ was presented. The stimulus disappeared when the monkey's direction of gaze moved off the stimulus, or after a maximum looking time of $5 \mathrm{~s}$. Each trial was followed by a $1 \mathrm{~s}$ intertrial interval. The VPLT was given in 51 daily blocks of 6,8 , or 10 trials each, chosen pseudorandomly, for a total of 400 trials each day. Each session, monkeys were presented with a total of 200 unique, complex stimuli. Each stimulus was presented twice during a given session, with up to eight intervening stimuli between successive presentations. A total of 9000 stimuli was used in this study.

Because the monkey controlled the duration of stimulus presentation, the duration of gaze on each stimulus provides a measure of the monkey's preference for the stimulus. We compared the amount of time the monkey spent looking at each stimulus during its first and second presentation. We designated the novel presentation of each stimulus the "encoding" phase and the repeated presentation the "recognition" phase of the task. Adult monkeys show a strong preference for novelty; therefore, a significant reduction in looking time between the two presentations of a stimulus indicated that the monkey had formed a memory of the stimulus and spent less time looking at the now familiar stimulus during its second presentation (Wilson and Goldman-Rakic, 1994). To control for varying interest in individual stimuli, recognition memory performance was calculated as the absolute change in looking time between presentations as a percentage of the amount of time the monkey spent looking at the first presentation of each stimulus.

Reward was not delivered during VPLT trials. However, five trials of the calibration task were presented between each VPLT block to give the monkey a chance to earn some reward and to verify calibration of the eye position. The number of trials in each VPLT block was varied to prevent the monkey from knowing when to expect the rewarded calibration trials.

\section{Electrophysiological recording methods}

The recording apparatus consisted of a multichannel microdrive (FHC) holding a manifold consisting of a single 23 gauge guide tube containing four independently moveable tungsten microelectrodes (FHC), with each electrode inside an individual polyamide tube. Electrode impedance was in the range of 1-2 M $\Omega$, and electrode tips were separated horizontally by $190 \mu \mathrm{m}$. For each recording, the guide tube was slowly lowered through the intact dura mater and advanced to $\sim 3.5 \mathrm{~mm}$ dorsal to the hippocampus using coordinates derived from the MRI scans. The electrodes were then slowly advanced out of the guide tube to the hippocampus. No attempt was made to select neurons based on firing pattern. At the end of each recording session, the microelectrodes and guide tube were retracted. All recordings took place in the anterior part of the left hippocampus. Recording sites were located in the CA3 field, dentate gyrus, and subiculum (supplemental Fig. 1, available at www.jneurosci. org as supplemental material).

Data amplification, filtering, and acquisition were performed with a multichannel acquisition processor (MAP) system from Plexon. The neural signal was split to separately extract the spike and the local field potential (LFP) components. For spike recordings, the signals were filtered from $250 \mathrm{~Hz}$ to $8 \mathrm{kHz}$, further amplified and digitized at $40 \mathrm{kHz}$. A threshold was set interactively, to separate spikes from noise, and spike waveforms were stored in a time window from $150 \mu$ s before to $700 \mu \mathrm{s}$ after threshold crossing. Each recording typically yielded two to six units; single units were sorted off-line using Off-line Sorter (Plexon). For LFP recordings, the signals were filtered with a passband of $0.7-170 \mathrm{~Hz}$, further amplified, and digitized at $1 \mathrm{kHz}$. Eye movement data were digitized and stored with a $240 \mathrm{~Hz}$ resolution.

\section{Data analysis}

All analyses were performed using custom programming in Matlab (Mathworks) and using FieldTrip (http://www.ru.nl/fcdonders/fieldtrip/), an open-source Matlab toolbox. To ensure that the monkeys had sufficient time to perceive the stimuli, analyses were limited to pairs of trials (corresponding to the two presentations of each stimulus) in which monkeys examined stimuli for at least $750 \mathrm{~ms}$ during the first presentation, which resulted in an average of 135 pairs of trials per session.

For each neuron, firing rate was calculated for the period including prestimulus fixation as well as stimulus presentation. Significant responses to stimuli were determined using Student's $t$ test to compare activity for the period from 100 to $500 \mathrm{~ms}$ after stimulus onset to a baseline period of $300 \mathrm{~ms}$ preceding stimulus onset. Only neurons judged to be visually responsive [i.e., those that displayed a significant mean firing rate modulation on the first (encoding) presentation] were included in additional analyses.

For the calculation of coherence and power spectra, the multitaper method was used to achieve optimal spectral concentration (Mitra and Pesaran, 1999; Jarvis and Mitra, 2001; Pesaran et al., 2002; Womelsdorf et al., 2006). Multitaper methods involve the use of multiple data tapers for spectral estimation. A $250 \mathrm{~ms}$ segment of data was multiplied by a data taper before Fourier transformation. A variety of tapers can be used, but an optimal family of orthogonal tapers is given by the prolate spheroidal functions or Slepian functions. For time length $T$ and bandwidth frequency $W$, up to $K=2 T W-1$ tapers are concentrated in frequency and suitable for use in spectral estimation. We used three Slepian tapers, providing an effective taper smoothing of $\pm 8 \mathrm{~Hz}$. For each taper, the data segment was multiplied with that taper and Fourier transformed, giving the windowed Fourier transform, $\tilde{x}_{k}(f)$

$$
\tilde{x}_{k}(f)=\sum_{1}^{N} w_{k}(t) x_{t} e^{-2 \pi i f t}
$$

where $x_{t},(t=1,2, \ldots, N)$ is the time series of the signal under consideration, and $w_{k}(t),(k=1,2, \ldots, K)$ are $K$ orthogonal taper functions. For spike signals, the firing rate was represented with a bin width of $1 \mathrm{~ms}$ and then subjected to spectral analysis like LFPs. 
The multitaper estimates for the spectrum $S_{x}(f)$ and the crossspectrum $S_{y x}(f)$ are given by the following:

$$
\begin{gathered}
S_{x}(f)=\frac{1}{K} \sum_{1}^{K}\left|\tilde{x}_{k}(f)\right|^{2} \\
S_{y x}(f)=\frac{1}{K} \sum_{1}^{K} \tilde{y}_{k}(f) \tilde{x}_{k}^{*}(f)
\end{gathered}
$$

Spectra and cross-spectra are averaged over trials before calculating the coherency $C_{y x}(f)$ as follows:

$$
C_{y x}(f)=\frac{S_{y x}(f)}{\sqrt{S_{x}(f) S_{y}(f)}}
$$

Coherency is a complex quantity. Its absolute value is termed coherence and ranges from 0 to 1 . A coherence value of 1 indicates that the two signals have a constant phase relationship (and amplitude covariation), and a value of 0 indicates the absence of any phase relationship. Thus, coherence is a measure of linear predictability that captures phase and amplitude correlations.

Coherence spectra were calculated between the spiking activity obtained on one electrode and LFP activity derived from a different electrode. Both coherence and power analyses were limited to LFPs derived from electrodes that also had isolated single units to ensure that LFPs were obtained from cell layers. We did not calculate coherence between LFPs and spiking activity obtained on the same electrode. This gave us a maximum of three spike-LFP coherence spectra for each neuron. Spikespike coherence spectra were also calculated between visually responsive neurons recorded within the same recording session, using the same methods described above for the calculation of coherence spectra between spiking activity and LFP activity.

\section{Correlating neuronal activity with memory performance}

Two methods were used to determine the relationship between neuronal activity and subsequent recognition memory performance. First, neuronal activity during encoding was compared for the stimuli that evoked the best and worst memory. The stimuli from each session were ranked in order of increasing recognition performance, quantified as the percentage change in looking time between first and second presentations for each stimulus. The 30 encoding trials with the lowest percentage change were designated "low recognition" and the 30 trials with the highest percentage change were designated "high recognition." After removing trials for which the looking time during the first stimulus presentation was $750 \mathrm{~ms}$ or less, 30 trials represented a median of $22.2 \%$ of all trials in the session. Comparisons between the two stimulus groups were made for neuronal firing rates, the evoked LFP response, spike and LFP power, spike-field coherence (SFC), and spike-spike coherence (SSC).

Neuronal firing rate. The visual response magnitude of each neuron was calculated across both groups of 30 trials from 100 to $500 \mathrm{~ms}$ after stimulus onset, expressed as a percentage of the baseline firing rate (such that a decrease in firing rate at stimulus onset assumed a negative value, and an increase in firing rate assumed a positive value). The absolute value of the percentage change value of each neuron was used to enable grouping of neurons with enhanced and depressed responses in the same analysis. Finally, a Student's $t$ test was used to determine whether the magnitude of the visual response was significantly different for high- and low-recognition trials across the population.

Evoked LFP. To compare stimulus-evoked LFPs across the two conditions, we calculated an average LFP across all LFPs time-locked to stimulus onset, for high-recognition and low-recognition trials. We then divided these signals into $10 \mathrm{~ms}$ bins and, using a Student's $t$ test, obtained a $p$ value for each bin. This allowed us to determine time points at which the two signals diverged significantly.

Spike and LFP spectra. Power spectra were calculated for each spike signal and all LFPs derived from electrodes that also had isolated single units, using the multitaper method (see details above). For spike spectra, neurons with enhanced firing rate responses to stimulus onset were analyzed separately from neurons with depressed firing rate responses. Cor- relations between spectra and recognition memory were tested using a nonparametric permutation test (see details below).

Spike-field coherence. To compare the average SFC across all neuronLFP pairs during encoding of high-recognition and low-recognition conditions, the frequency range within the $30-100 \mathrm{~Hz}$ gamma band for which each neuron-LFP pair showed the highest SFC at 100-400 ms after stimulus onset across all encoding trials was identified (average frequency window size was $21.4 \pm 0.7 \mathrm{~Hz}$ ). SFC was calculated within this frequency window across all high-recognition and low-recognition trials, separately for each pair. Then, these values were averaged across all neuron-LFP pairs.

To test for statistical significance of differences between spectra during the high-recognition and low-recognition conditions, we performed a nonparametric permutation test, with the median difference between conditions as our test statistic. The test involves a comparison of the observed difference against a reference distribution of differences under the null hypothesis of no significant modulation of the spike or LFP power or SFC at individual frequencies between conditions. The reference distribution was obtained by performing the following procedure 10,000 times. For each recording site (or pairs of sites), a random decision was made to which condition the data from either condition was assigned. We then calculated the test statistic at each frequency for these randomly assigned conditions and stored only the minimal and maximal difference across frequencies. From the resulting distribution of 10,000 minimal and maximal differences, we determined the 2.5 th and the 97.5th percentile. The empirically observed, nonrandomized difference at a particular frequency was considered statistically significant $(p<$ 0.05 ), when it was larger than the 97.5th or smaller than the 2.5th percentile of the reference distribution. This procedure corresponds to a two-sided test with a global false-positive rate of $5 \%$ and correction for the multiple comparisons across frequencies (Nichols and Holmes, 2002; Maris and Oostenveld, 2007). We used this nonparametric permutation approach, because (1) it is free of assumptions about the underlying distributions, (2) it is not affected by partial dependence among the time-frequency tiles, and (3) it allows for correction for multiple comparisons without additional assumptions.

Additionally, we identified neuron-LFP pairs showing significant gamma-band coherence using the following method. To test the significance of coherence values, we calculated the time-averaged coherence across the time period of 100-400 ms after stimulus onset for each pair, and then transformed these values to $Z$ scores using the following formula:

$$
Z=\operatorname{arctanh}(\sqrt{c}) \times \sqrt{2 L}
$$

where $C$ is the coherence value, and $L$ is the number of independent estimates (Rosenberg et al., 1989; Kilner et al., 2000). Z-transformed coherence values were thus calculated for each neuron-LFP pair, across all high-recognition trials (novel presentations of the 30 stimuli from each session for which the monkey subsequently showed the best recognition). We considered a pair to have significant spike-field coherence if this $Z$-transformed gamma-band coherence value was $>2$ for at least five consecutive frequency values (spanning $16.6 \mathrm{~Hz}$ ).

Spike-spike coherence. Our methods for analyzing the relationship between SSC for visually responsive neuron pairs and behavior are identical with the analyses applied to SFC data, as described above. Specifically, the $Z$-transformed coherence values were used to determine neuron-neuron pairs with significant gamma-band coherence, and the nonparametric permutation test described above was used to determine whether spikespike coherence was significantly correlated with memory performance.

\section{Correlations with memory and attention: binning analysis}

The second analysis we performed to determine the relationship between neuronal activity and performance considered correlations on a trial-bytrial basis. For each recording session, encoding trials were sorted in two ways: in terms of increasing percentage change in looking time between the encoding trial and the subsequent repetition of the stimulus (recognition memory performance), and in terms of total looking time for the encoding trial (attention). For each measure, 10 bins of 30 trials each were defined, with bin centers spaced at equivalent intervals. An equal 
number of trials per bin was used to avoid sample size biases. As a consequence, in some cases, this resulted in slightly overlapping bins and a few trials that were not included in any bin. For each neuron-LFP pair, the frequency range for which the pair showed the highest SFC at $100-$ $400 \mathrm{~ms}$ after stimulus onset across all encoding trials was identified, and then the SFC in that frequency range at 100-400 ms was calculated, separately for each bin, across the 30 trials in each bin. Finally, the correlation between the 10 bins of each task parameter value (either recognition memory performance or attention) and coherence during encoding was calculated. Across pairs of recording sites, this resulted in a population of correlation coefficients and slopes for each measure. A sign test was performed on each distribution to determine statistical significance.

For the stimulus-evoked LFP, this analysis was performed in the way described above with one difference: for each bin of trials, we averaged the LFP amplitude from 270 to $570 \mathrm{~ms}$ after stimulus onset for novel trials (the time during which there was a significant difference in the LFP amplitude between high-recognition and low-recognition trials across all recorded LFPs). The slope and the correlation coefficient were calculated for this trial-averaged LFP amplitude across all bins, separately for each LFP. A sign test was then performed on each distribution of slopes and correlation coefficients.

\section{Correlations with time within session:} binning analysis

To determine possible changes in behavior or neuronal activity that may have occurred within the session, for each recording session, all 200 stimuli were organized into the order in which they were presented within each session. Ten bins of 20 stimuli each were then defined, and five measures were calculated for each bin: the mean percentage change in looking time from the first to the second presentation (recognition memory performance); the absolute looking time during novel stimulus presentation; the firing rate modulation, defined as the absolute value of the change in firing rate from the $300 \mathrm{~ms}$ preceding stimulus onset to the time period $100-500 \mathrm{~ms}$ after stimulus onset; gamma-band SFC from 100 to $400 \mathrm{~ms}$ after stimulus onset, using the same frequency window as that used in the binning analysis described above; and LFP amplitude averaged over the time period of $270-570 \mathrm{~ms}$ after stimulus onset for each novel stimulus presentation.

\section{Results}

\section{Behavioral results}

Figure $1 B$ depicts an example of the monkey's eye movements during the first (yellow trace) and second (red trace) presentations of a stimulus in the VPLT. In this example, and across the majority of trials, the monkey spent more time looking at a stimulus when it was novel compared with when it was repeated. Across 45 sessions, the monkeys demonstrated robust recognition memory performance. There was a significant $(p<0.001)$ decrease in looking time for the repeated presentation (average looking times for novel and repeat trials were 2.7 and $0.8 \mathrm{~s}$, respectively). The median reduction in looking time was $70.7 \%$ (67.3\% in monkey A and $72.8 \%$ in monkey B) (Fig. 1C).

\section{Neuronal activity in the hippocampus}

We recorded spikes from 131 isolated single neurons (67 in monkey A and 64 in monkey B, respectively) as well as LFPs in the hippocampal formation in two rhesus monkeys performing the VPLT. Eighty-six neurons (66\%) gave a significant response to the first (encoding) presentation of stimuli, with either enhanced (34 neurons) or depressed (52 neurons) responses compared with baseline (Fig. $2 A$; supplemental Table 1, available at www. jneurosci.org as supplemental material). Consistent with recent findings from human epileptic patients (Rutishauser et al., 2006), a substantial proportion of these visually responsive units (36\%) showed a modulation in firing rate based on stimulus novelty.

Neuronal synchronization during the encoding phase of the task was assessed by calculating SFC between each visually responsive neuron and the LFP recorded simultaneously on a separate electrode ( $n=175$ neuron-LFP pairs). The LFP results from the extracellular current flow that corresponds primarily to the summed postsynaptic potentials from the dendritic fields of local cell groups (Buzsáki, 2004). Thus, SFC is a measure of linear predictability that captures phase and amplitude correlations between neuronal input (LFP) and output (spiking activity). SFC typically increased on visual stimulation, and these increases were most prominent in the $1-8 \mathrm{~Hz}$ range (delta/theta band), and the $30-100 \mathrm{~Hz}$ range (gamma band). Coherence of $<20 \mathrm{~Hz}$ was not significantly modulated by recognition memory performance on the VPLT. Accordingly, we have confined our analysis and discussion to neuronal synchronization in the gamma band.

Across the population gamma-band SFC tended to cluster in one of two frequency bands: low gamma $(30-60 \mathrm{~Hz})$ (Fig. $2 \mathrm{~B}, \mathrm{C}$, bottom) and high gamma $(60-100 \mathrm{~Hz})$ (Fig. $2 B, C$, top). Of 86 neurons, 42 displayed a range of coherence that included coherence in the $60 \mathrm{~Hz}$ band. However, not all those neurons necessarily showed coherence centered around $60 \mathrm{~Hz}$ : 20 neurons displayed a band of increased coherence with $60 \mathrm{~Hz}$ as either the 
A
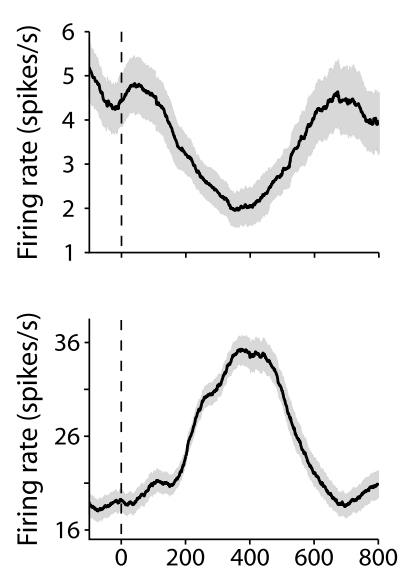

Time from stimulus onset $(\mathrm{ms})$
B
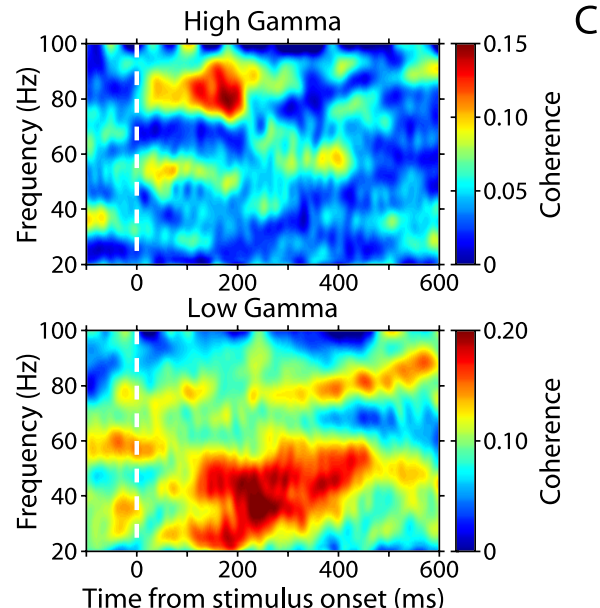

$C$
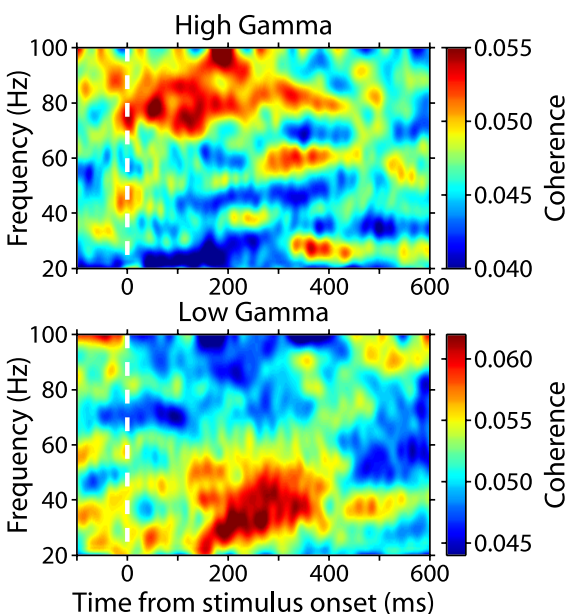

Figure 2. Firing rate and spike-field coherence during stimulus encoding. $\boldsymbol{A}$, Average firing rates for two example hippocampal neurons during encoding. Shaded areas represent SEM. $\boldsymbol{B}$, Spike-field coherence as a function of time ( $\boldsymbol{x}$-axis) and frequency ( $\boldsymbol{y}$-axis) during all encoding trials for the two example neurons shown in $\boldsymbol{A}$; coherence was calculated between the neuron on one electrode and the LFP recorded on a separate electrode. C, Coherence as a function of time and frequency averaged across all encoding trials for high-gamma (top) and low-gamma (bottom) neurons.

upper or lower limit and thus had a substantial portion of increased coherence either above or below $60 \mathrm{~Hz}$. Of the remaining 22 neurons, only 4 showed peak coherence at $60 \mathrm{~Hz}$. For these neurons, we designated each as high or low gamma based on the entire frequency band in which the neuron showed coherence during encoding trials, and whether the bulk of this frequency band lay above or below $60 \mathrm{~Hz}$. Using this method, 3 neurons were designated as high gamma and 1 was designated as low gamma. There was no significant relationship between the peak frequency of gamma-band coherence and the response properties of neurons: 59\% of neurons with enhanced firing responses to stimuli exhibited coherence in the low gamma range, whereas $56 \%$ of neurons with depressed firing responses to stimuli exhibited coherence in high gamma $(p>0.10)$ (supplemental Table 1 , available at www.jneurosci.org as supplemental material).

We additionally analyzed all neuron-LFP pairs with visually responsive single units to determine how many pairs exhibited significant SFC during the initial presentation of subsequently well recognized stimuli. Of these neuron-LFP pairs, 151 (86\%) met the criterion we set for significant gamma-band SFC. Additionally, of 83 pairs of simultaneously recorded visually responsive neurons, 54 pairs (65\%) showed significant gamma-band SSC during high-recognition trials.

\section{Hippocampal gamma-band synchronization reflects recognition memory performance}

Figure 3, $A$ and $B$, depicts the firing rate and SFC for highrecognition and low-recognition trials for an example recording pair. For this example neuron, and across the population, firing rates during encoding were not significantly modulated by subsequent recognition memory performance $(p>0.05)$ (Fig. $3 A)$. In contrast, for this example (Fig. $3 B$ ) and across the population (Fig. 3C-F), gamma-band coherence was enhanced during the encoding of stimuli that were subsequently well recognized relative to those stimuli that were poorly recognized.

Increases in SFC during the presentation of novel stimuli usually covered limited frequency bands within the broader gammaband range. This tendency of spikes to lock coherently with LFPs in a narrow band of a particular gamma frequency has also been reported in the rodent neocortex (Sirota et al., 2008). For this reason, we identified a separate frequency range for each neuron to analyze changes in SFC with respect to memory (see Materials and Methods). Figure $3 C$ shows the average recognition-related modulations in coherence across the population of recording sites. High recognition represents the gamma-band SFC during the encoding of the 30 stimuli in each session with the best subsequent recognition, and low recognition corresponds to the 30 stimuli with the worst recognition. Across the population, there was a $\sim 10 \%$ increase in gamma-band synchronization during encoding of stimuli that were subsequently well recognized relative to those stimuli that were poorly recognized. This enhancement reached significance beginning $120 \mathrm{~ms}$ after stimulus onset.

Average SFC for the two memory conditions are displayed separately for spike-field pairs displaying high- $(>60 \mathrm{~Hz}$ peak frequency) (Fig. 3D) and low-gamma synchronization $(<60 \mathrm{~Hz})$ (Fig. 3E) (for details, see supplemental methods, available at www.jneurosci.org as supplemental material). The results of the nonparametric permutation analysis revealed that gamma-band coherence was significantly enhanced during high-recognition trials as early as $100 \mathrm{~ms}$ after stimulus onset for high-gamma spike-field pairs ( $p<0.05$, corrected for multiple comparisons) (Fig. 3F, top panel). Although there was a strong trend for enhanced gamma-band coherence across the low gamma spikefield pairs, this did not reach statistical significance (Fig. 3F, bottom panel). This may have been attributable to a lack of sensitivity because the same analysis using multiunit activity revealed significant memory-related modulations in gamma-band coherence for both high-gamma and low-gamma pairs (supplemental Fig. 2, available at www.jneurosci.org as supplemental material). Because the sensitivity of coherence measures are proportional to the number of neurons contributing to the analysis, coherence analyses of single-unit activity are less sensitive than analyses of multiunit activity (Zeitler et al., 2006). Therefore, it is possible that single-unit spike-field coherence did not reach significance for the low-gamma group because of a loss in sensitivity compared with the multiunit analysis.

We also tested whether SSC was significantly correlated with memory performance using the nonparametric permutation test. Each neuron-neuron pair showing significant coherence was designated as "high gamma" or "low gamma" based on the frequency band in which coherence across all novel stimulus presentations increased in the time period of 100-400 ms after stimulus onset. We applied the permutation test to each group of pairs separately: a small, but significant, cluster of SSC was seen 
A

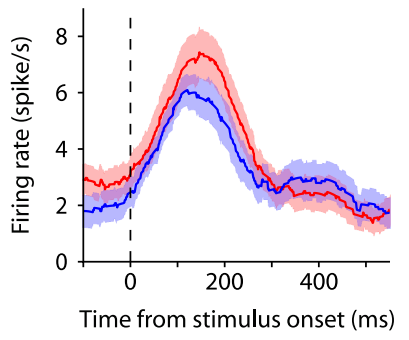

$\mathrm{D}$

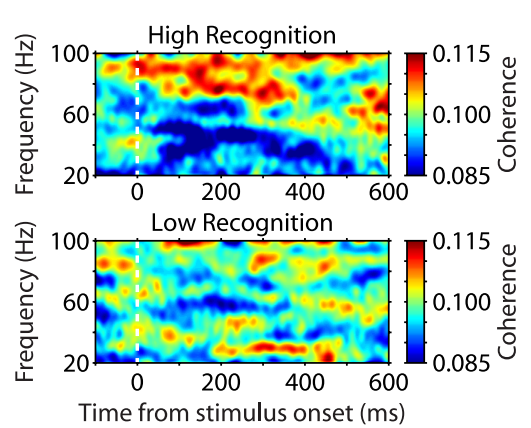

B

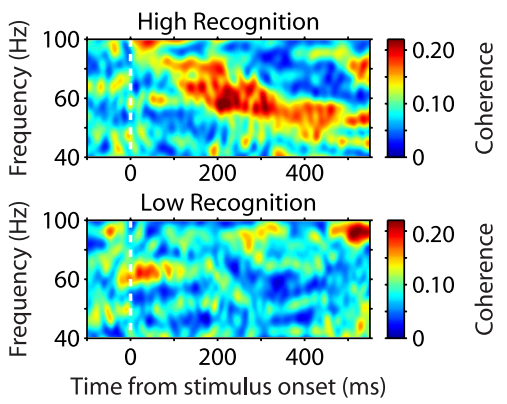

$\mathrm{E}$

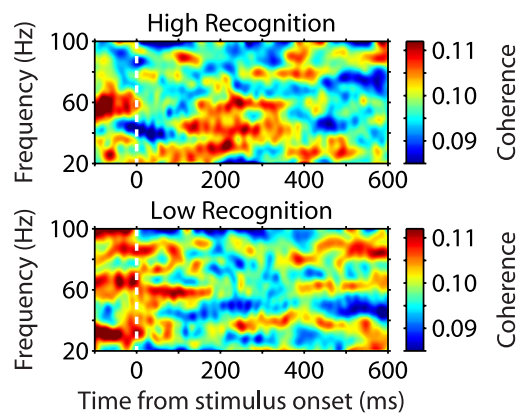

C

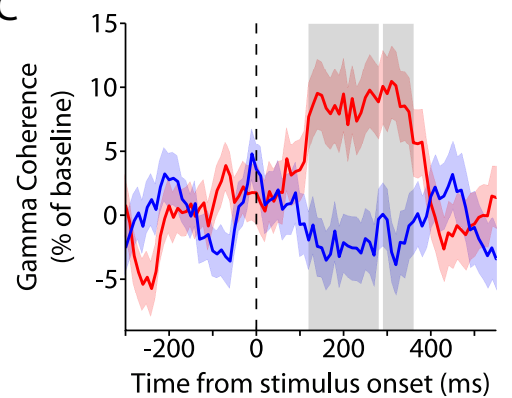

$\mathrm{F}$

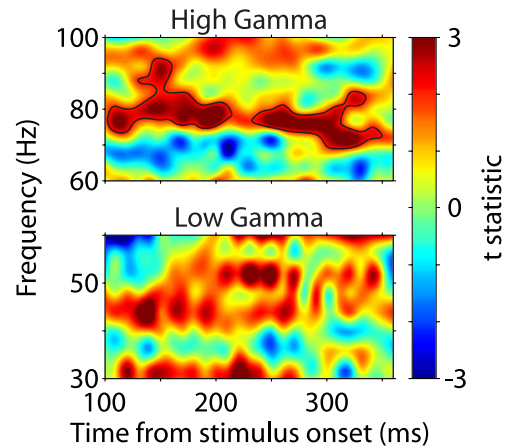

Figure 3. Gamma-band spike-field coherence during stimulus encoding predicts subsequent recognition. A, Average firing rate of an example hippocampal neuron for high-recognition (red) and low-recognition (blue) trials. There was no difference in firing rate across conditions. The red and blue shaded areas represent SEM. $\boldsymbol{B}$, Coherence as a function of time and frequency between the example neuron in $A$ and the LFP recorded on a separate electrode, for high-recognition (top) and low-recognition (bottom) trials. Coherence (52- $68 \mathrm{~Hz}$ ) was significantly enhanced during the encoding of subsequently well recognized stimuli. C, Gamma-band coherence expressed as percentage of baseline averaged over 175 hippocampal recording pairs, during high-recognition (red) and low-recognition (blue) trials, as a function of time from stimulus onset. The red and blue shaded areas represent SEM. The gray shaded area represents time points at which gamma-band coherence was significantly different for the two conditions $(p<0.01)$. $\boldsymbol{D}$, Coherence averaged across all high-gamma neurons, for high-recognition (top) and low-recognition (bottom) trials. $\boldsymbol{E}$, Same as $\boldsymbol{D}$, but for low-gamma neurons. $\boldsymbol{F}$, Modulation of coherence between high-recognition and low-recognition trials, for high gamma (top) and low gamma (bottom). Areas of significant coherence modulation are outlined in black (nonparametric randomization test, corrected for multiple comparisons across time and frequency).

for the high-gamma pairs $(n=32)$ but not for the low-gamma pairs $(n=22)$. This result is presented in supplemental Figure 3 (available at www.jneurosci.org as supplemental material).

\section{Relationship between gamma-band synchronization and behavior: memory versus attention}

It is important to consider whether the observed synchronization among hippocampal neurons primarily reflects successful memory encoding or the attentive state of the animal. Increased attention to a stimulus likely leads to more successful memory encoding and may cause enhanced neuronal synchronization among hippocampal neurons. With 200 novel stimuli in each recording session, we have to assume that some stimuli are more interesting to the monkey and attract the monkey's attention more than other stimuli. Because the task design allows the monkey to determine the length of stimulus presentation by continuing to look at or looking away from each stimulus, we take as an assumption that the length of looking time for the initial presentation of a stimulus (encoding) reflects the animal's interest in, and attention to, the stimulus. Although other factors may influence looking time in isolated instances (e.g., the monkey's distractibility), over many trials, the monkey's interest in and attention to the stimulus is most likely the overriding factor in determining looking time during novel presentation. If hippocampal synchronization reflects primarily attentive mechanisms, increasing gamma-band coherence in the hippocampus would correlate with increasing length of time spent looking at novel stimuli. To quantify the extent to which neuronal synchronization among hippocampal neurons correlated with recognition memory and attention, for each recording session, we organized all encoding trials into bins, either by increasing recognition memory performance (expressed as the percentage change in looking time) or increased attention (expressed as the duration of looking time during the encoding phase). We then correlated the magnitude of spike-field coherence and the behavioral measures of recognition memory performance and attention, as described in Materials and Methods. For the example neuron-LFP pair depicted in Figure 4A, gamma-band spike-field coherence was significantly correlated with recognition memory performance $(p<0.005)$ (Fig. $4 A$, left) but not with attention $(p>$ $0.10)$ (Fig. $4 A$, right). Across the population, the correlation coefficients and the slopes for all neuron-LFP pairs displayed a significant positive distribution $(p<0.001)$ (Fig. $4 B, C$, left) for recognition memory performance, but not for attention $(p>$ $0.10)$ (Fig. $4 B, C$, right). A consistent result was obtained when the multiple spike-field coherence results for each single unit were averaged (supplemental Fig. 4, available at www.jneurosci. org as supplemental material). These data suggest that the attentive state of the animal during encoding, as indexed by duration of looking, does not explain the effects of hippocampal gammaband synchronization on recognition memory performance.

Previous studies have found that principal cells and interneurons play different roles in the generation of gamma-band oscillations in the hippocampus (Bragin et al., 1995; Chrobak and Buzsáki, 1996; Csicsvari et al., 2003). We categorized neurons as putative principal cells or putative interneurons, taking into consideration both the average firing rate during the fixation period preceding stimulus onset and the width of spike waveforms. Both populations of neurons displayed significant gamma-band spikefield coherence modulations during stimulus encoding that pre- 
A

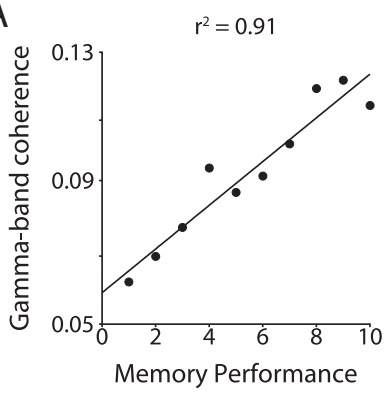

B

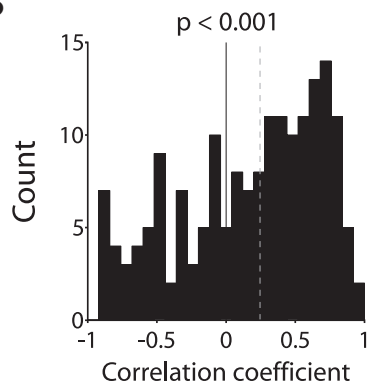

C

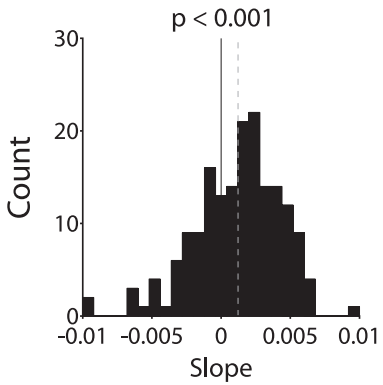

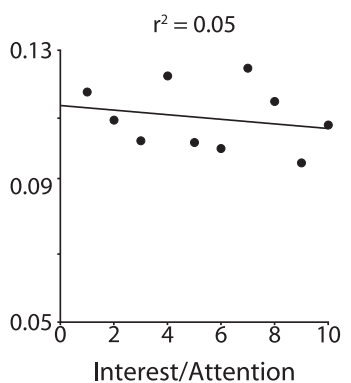
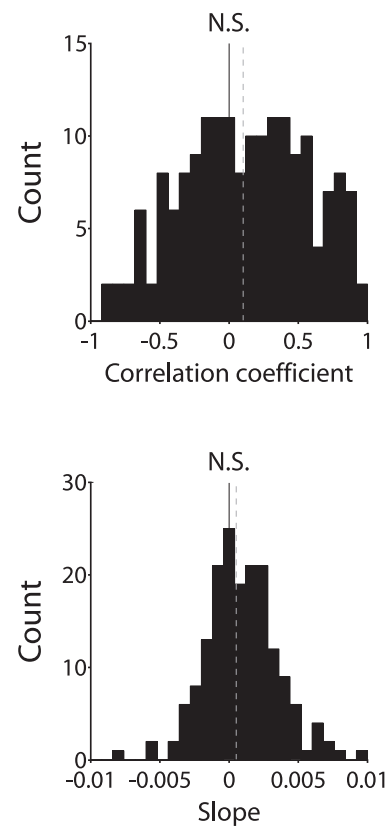

Figure 4. Coherence is correlated with recognition memory, but not with attention. A, Gamma-band spike-field coherence for one example neuron-LFP pair, binned according to percentage change in looking time (left) or looking time during encoding (right). The line indicates linear fit to data. $\boldsymbol{B}$, Histograms depicting correlation coefficients between gammaband spike-field coherence and behavior across all neuron-LFP pairs when binned according to percentage change in looking time (left) or looking time during encoding (right). The black line indicates zero; the dashed gray line indicates median. $\boldsymbol{C}$, Same as $\boldsymbol{B}$, but for slopes. The dashed gray line indicates median.

dicted subsequent recognition memory (data not shown). Of the 76 visually responsive putative pyramidal cells, 39 were classified as high gamma and 37 as low gamma. Ten neurons were classified as putative interneurons, four of which were designated high gamma. Accordingly, the data do not suggest that the high-versus low-gamma classification was correlated with cell type.

Along with coherence, we also derived power spectra for all LFPs and spike trains. There was no significant effect of memory performance on power in the spike spectra across the population (data not shown). However, LFP power from 40 to $65 \mathrm{~Hz}$ was significantly enhanced during the encoding of well remembered stimuli compared with the encoding of poorly remembered stimuli $\sim 80-300 \mathrm{~ms}$ after stimulus onset (Fig. 5). Although the gammaband power effects occurred at the same time as the effects in gamma-band coherence, it is important to note that spike-field coherence is normalized by power in both the spike spectrum and the LFP spectrum (see Materials and Methods). In other words, coherence represents the consistency of the phase relationship between the single unit rhythm and the LFP rhythm, regardless of the power in either rhythm. Thus, although both signals are cor-

A

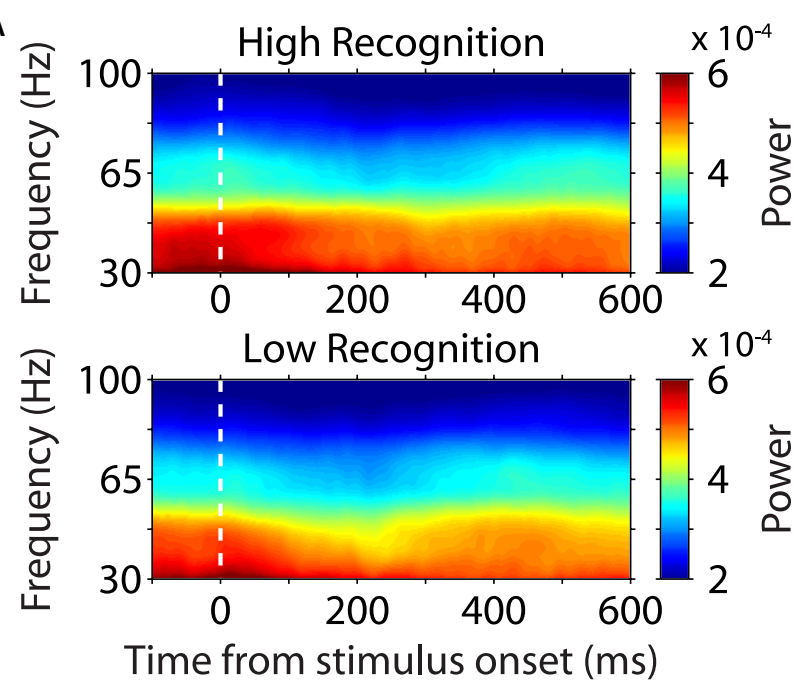

B

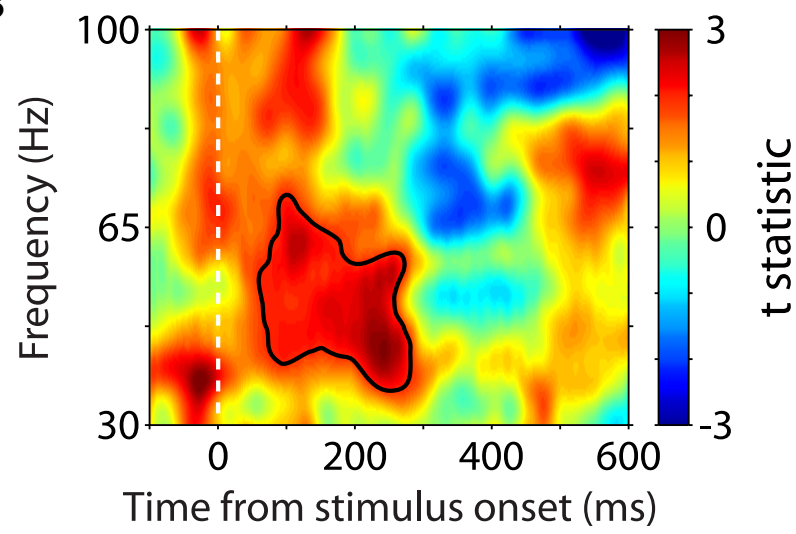

Figure 5. Gamma-band LFP power during stimulus encoding predicts subsequent recognition. $A$, Gamma-band power averaged across all LFPs during the encoding of high-recognition (top) and low-recognition (bottom) stimuli. LFP spectra have been normalized by $1 / f$ for visualization. B, Modulation of gamma-band power between high-recognition and lowrecognition stimuli. The area of significant power modulation is outlined in black (nonparametric randomization test, corrected for multiple comparisons across time and frequency).

related with the strength of memory encoding, each represents a distinct neural mechanism.

The results from the nonparametric permutation test revealed significant differences between memory conditions for spike-field coherence, spike-spike coherence, and gamma-band power after stimulus onset. However, there were also small clusters of increased gamma-band coherence against background activity before stimulus onset (Fig. $3 D, E$ ). An additional permutation test applied to the baseline period before stimulus onset revealed no clusters of significant prestimulus modulations for either single-unit SFC (supplemental Fig. 5, available at www.jneurosci.org as supplemental material), multiunit SFC (supplemental Fig. $2 A$, available at www. jneurosci.org as supplemental material), single-unit SSC (supplemental Fig. $3 A$, available at www.jneurosci.org as supplemental material), or gamma-band power (supplemental Fig. 6, available at www.jneurosci.org as supplemental material). Therefore, unlike the stimulus-related activity, none of the prestimulus activity we recorded was modulated by recognition memory performance.

\section{Relationship between local field potential and behavior}

There have been a number of studies investigating neural activity during presentation of novel or rare stimuli in humans and mon- 
A

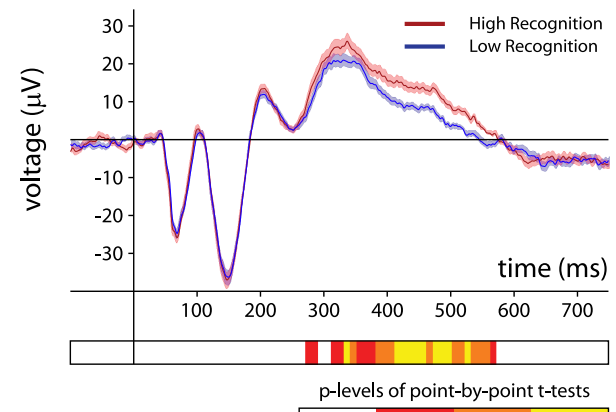

p-levels of point-by-point t-tests
n.s. $\quad<0.01<0.001<0.0001$

B
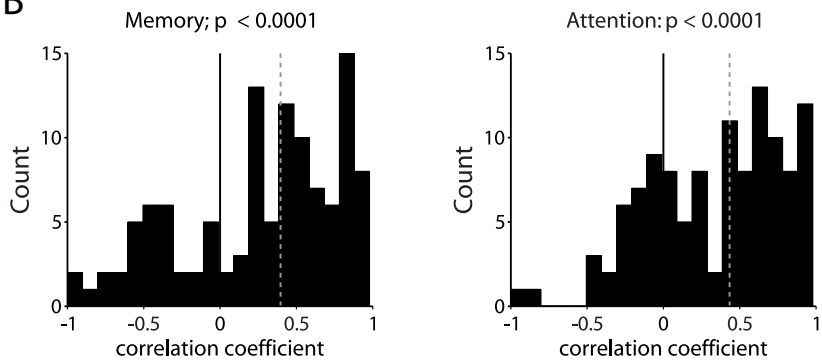

C
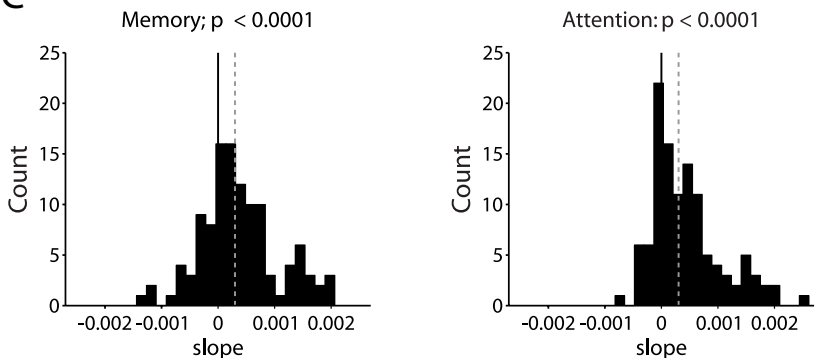

Figure 6. Stimulus-evoked LFP is modulated by both attention and recognition memory. $\boldsymbol{A}$, Stimulus-evoked modulations in LFP amplitude averaged across 114 LFPs during encoding of highrecognition (red) and low-recognition (blue) stimuli. The shaded areas represent SEM. Values of $p$ for significance tests at each consecutive $10 \mathrm{~ms}$ time bin are shown in the color plotbelow the graph. Time bins shown in yellow represent values of $p<0.0001$. $\boldsymbol{B}$, Histograms depicting correlation coefficients of the linear functions fit to LFP data across all LFPs when binned according to percentage change in looking time (left) or looking time during encoding (right). The black line indicates zero; the dashed gray line indicates median. $\boldsymbol{C}$, Same as $\boldsymbol{B}$, but for slopes. The dashed gray line indicates median.

keys. One of the most well characterized components of this neural response, the P300 component of the event-related potential, is thought to represent the conscious processing, or encoding, of such stimuli (Soltani and Knight, 2000; Polich, 2007). The MTL-P300, recorded via depth electrodes in humans, is a locally generated version of the P300 associated with the hippocampal contribution (Halgren et al., 1998; Fell et al., 2004). Figure 6A depicts the average stimulus-evoked LFP aligned to stimulus onset for high-recognition and low-recognition trials, averaged across all 114 LFPs. There was a significant divergence in the signal as early as $270 \mathrm{~ms}$ after stimulus onset that predicted subsequent recognition memory performance. We analyzed the magnitude of the stimulus-evoked LFP with respect to memory performance and attention throughout the session using a binning analysis, similar to our previous analysis for spike-field coherence. There was a significant positive relationship between LFP amplitude during stimulus encoding and subsequent recognition memory performance, as well as between LFP amplitude and looking time during encoding (Fig. 6B,C). These data suggest that, unlike gamma-band coherence, changes in the LFP amplitude reflect both attention and memory on a trial-by-trial basis, which is consistent with previous studies associating the P300 with attentional processing (Kok, 2001) and hippocampal-dependent processing of novel stimuli (Knight, 1996). Interestingly, this P300-like effect did not begin until nearly $170 \mathrm{~ms}$ after the earliest effects seen in gamma-band spike-field coherence.

\section{Additional behavioral controls}

We also considered the possibility that changes in behavior or neuronal activity through the recording session may affect the interpretation of these results. On average, the monkeys required 58 min to complete the session, viewing two presentations of each of 200 stimuli. It is possible that the stimuli presented at the beginning and end of the session evoked different neuronal responses. It is also possible that the monkey experienced fatigue through the session that influenced his performance. To address this issue, we analyzed memory performance, stimulus-evoked firing rates, and the magnitude of SFC with respect to time within the recording session. One-way ANOVAs revealed that there was no significant relationship between time within the session and recognition memory $\left(F_{(9,439)}=0.57 ; p>0.1\right)$ (Fig. 7$)$, absolute looking time during novel stimulus presentation $\left(F_{(9,437)}=0.39\right.$; $p>0.1)($ Fig. $7 A)$, firing rate modulation $\left(F_{(9,850)}=0.28 ; p>0.1\right)$ (Fig. $7 B)$, or gamma-band $\operatorname{SFC}\left(F_{(9,1740)}=0.96 ; p>0.1\right)$ (Fig. $7 C)$. However, there was a significant negative correlation between LFP amplitude and the time course of the recording session $\left(F_{(9,1120)}=2.22 ; p<0.05\right)$ (Fig. $\left.7 D\right)$. Because this was the only measure that showed any significant correlation with time within the session, it is unclear whether this decline in LFP amplitude is related to fatigue, or some other mechanism.

Additionally, we determined the amount of time required to achieve fixation before each stimulus presentation. An increased time to achieve fixation would indicate that the monkey's attention or arousal level had declined. Over all 45 recording sessions, we found that that there was no significant difference in this measure between high-recognition and low-recognition trials $(p>0.10)$. These data suggest that fluctuations in general alertness or arousal levels are not correlated with modulations in gamma-band synchronization in the hippocampus.

\section{Discussion}

Our findings show that spikes from isolated single units in the hippocampus are phase locked to each other and to gamma-band oscillations in simultaneously recorded hippocampal LFPs during memory encoding. Furthermore, the magnitude of this phase locking is correlated with subsequent recognition memory performance. These results suggest that memory encoding is accompanied by enhanced coordination between hippocampal neurons.

Fell et al. (2001) previously showed that successful recognition memory encoding is correlated with increased gamma-band synchronization between local EEG oscillations in the hippocampus and rhinal cortex of human epileptic patients. The current findings extend these observations to hippocampal neurons, indicating that single units within the hippocampus synchronize the timing of their spikes to the local network oscillations during memory formation, perhaps as a mechanism by which neurons sharing similar response properties might undergo functional coupling. We also found that gamma-band power in hippocampal LFPs during encoding is significantly correlated with subsequent recognition memory performance. These results are consistent with studies in human epileptic patients that have associated changes in hippocampal gamma-band oscillations with memory (Sederberg et al., 2007a,b). Similar observations have been made in 
A
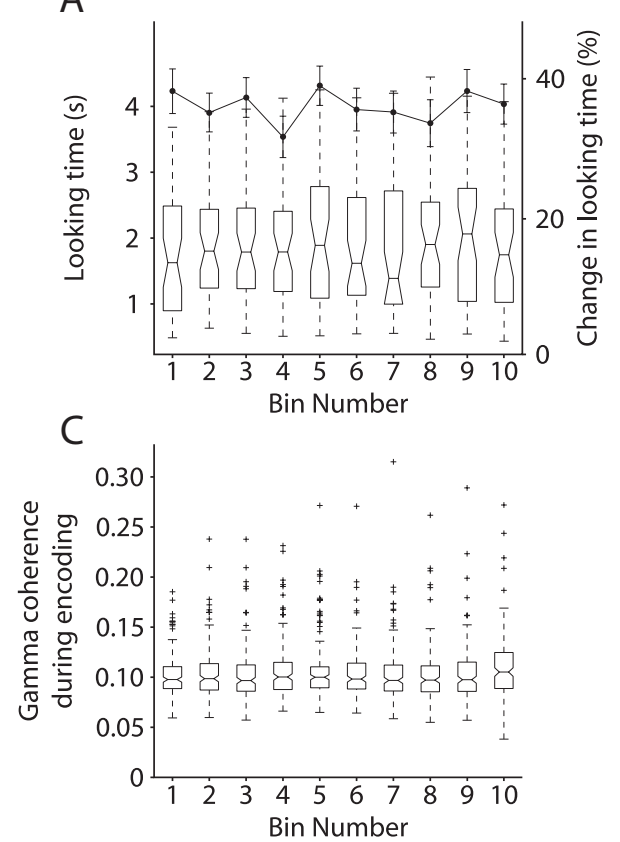

B

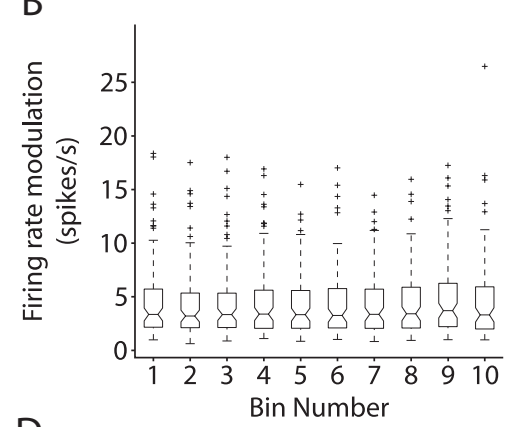

D

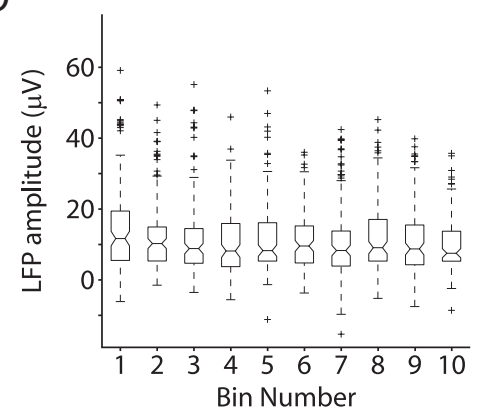

Figure 7. Behavioral and neural measures as a function of time within session. $A$, Box plot, Absolute looking time during novel stimulus presentation, averaged within bins of 20 trials each, across recording sessions. There was no significant effect of bin number on looking time. Line plot, Percentage change in looking time averaged within bins of 20 trials each, across all recording sessions. There was no significant effect of bin number on behavior across sessions. $\boldsymbol{B}$, Magnitude of the response for all visually responsive neurons, averaged within bins of 20 trials each, across recording sessions. There was no significant effect of bin number on firing rate across neurons. C, Gamma-band spike-field coherence for all visually responsive neurons, averaged within each bin, across recording sessions. There was no significant effect of bin number on coherence across neuron-LFP pairs. D, LFP amplitude across the $270-570 \mathrm{~ms}$ period after novel stimulus onset for all LFPs, averaged within each bin, across recording sessions. There was a significant negative correlation between LFP amplitude and the time course of the recording session (one-way ANOVA, $\left.F_{(9,1120)}=2.22 ; p<0.05\right)$.

monkey parietal cortex in relation to working memory (Pesaran et al., 2002). In that study, both power and coherence in the gamma band were elevated during the delay period of a working memory task. Together, these findings suggest that synchronization between spiking activity and oscillatory field activity may be an important mechanism for holding a representation of behaviorally relevant stimuli "on-line."

Previous studies in rodents have linked hippocampal gammaband synchronization to memory processes (Robbe et al., 2006; Montgomery and Buzsáki, 2007). In these studies, both the power and the coherence of gamma-band oscillations in hippocampal LFPs were enhanced in relation to the cognitive demands of a hippocampal-dependent task. Consistent with the current study, it was shown that modulations in neuronal synchronization can be dissociated from modulations in firing rate (Robbe et al., 2006), further supporting the notion that changes in the temporal structure of neuronal activity may affect computational outcomes. The current study extends these findings by showing a direct relationship between hippocampal gamma-band coherence and recognition memory performance.

How might gamma-band synchronization in the hippocampus improve encoding? By ensuring that the activity of multiple neurons is correlated within short (i.e., $10 \mathrm{~ms}$ ) temporal windows, gamma-band synchronization could underlie the transient formation of functional neuronal ensembles (Fries, 2005; Womelsdorf et al., 2007). For example, a population of neurons may respond to a particular stimulus by synchronizing its firing in the gamma range, and this may contribute to spike timing-dependent long-term potentiation (Bi and Poo, 1998), thereby strengthening the

connections between these neurons. Gamma-band synchronization among hippocampal neurons may also serve to enhance the impact of hippocampal neurons on output targets in the entorhinal cortex. For example, gamma-band synchronization may result in increased temporal summation of synaptic input on neurons downstream of hippocampal ensembles, thereby increasing the likelihood that these neurons will fire. Such a mechanism would in turn enhance the relay of memory signals to higher-order sensory areas and other areas important for memory storage. Although the difference in average coherence measures between recognition memory conditions is small, evidence from computational studies suggests that small increases in even weakly correlated inputs to neurons can cause substantial increases in the probability of firing of downstream neurons (Salinas and Sejnowski, 2000).

One caveat is that spike-field coherence does not directly reflect synchronization in the signals being projected to downstream areas, but only implies such an interaction, assuming that some component of the output is reflected in the LFP. Although the results of our analysis of spike-spike coherence provide evidence for synchrony among hippocampal units, future studies are needed to provide a more direct measurement of the degree to which synchronization within the hippocampus affects changes in the activity of downstream targets (e.g., with simultaneous recordings in the hippocampus and the entorhinal cortex).

It is important to consider the extent to which memory effects can be dissociated from attentional effects in assessing performance on the VPLT. Although these processes cannot be completely dissociated with this behavioral task, there is evidence from previous studies that memory and attention depend on different brain regions. In particular, the finding that monkeys with hippocampal lesions (Zola et al., 2000) and amnesic patients (McKee and Squire, 1993) display intact novelty preference as long as the delay between first and second stimulus presentation is short ( 1 and $0.5 \mathrm{~s}$, respectively) but are impaired with increasing delays (10 s and longer) supports this idea. At the same time, increased attention during stimulus presentation may lead to better subsequent memory. This could result in neural signals that underlie both processes covarying with behavioral performance. Our data suggest that gamma-band coherence in the hippocampus more reliably predicts successful recognition memory performance than increased attention to stimuli. In contrast, the stimulus-evoked LFP in the hippocampus appears to reflect both memory encoding and attentional processes.

To our knowledge, this study is unique in its separation of gamma-band oscillations recorded in the primate hippocampus into high and low gamma. A number of recent studies have observed oscillatory synchrony in either high or low gamma in other brain regions, and in many cases these frequency bands have been associated with distinct aspects of cognition (Edwards et al., 2005; Hoogenboom et al., 2006; Wyart and Tallon-Baudry, 2008, 2009). 
Cortical oscillations in the high gamma band tend to exhibit higher phase-locking with theta oscillations in humans (Canolty et al., 2006) and rodents (Sirota et al., 2008). Additionally, oscillations in the high gamma-band range have been associated with the hemodynamic response measured using blood oxygen leveldependent functional MRI (Niessing et al., 2005).

In the current study, the results of the nonparametric test for single units revealed a significant difference across memory conditions only for the high-gamma neurons. However, analyses including both high- and low-gamma neurons revealed significant differences between successful (high-recognition) and less successful (low-recognition) encoding (Fig. 3C), as well as a significant positive correlation between trial-by-trial modulations in coherence and recognition memory (Fig. 4). Nevertheless, these different populations of neurons may make distinct contributions to behavior through their participation in different modes of network activity.

Visual stimuli induced a clear increase in gamma-band synchronization that was associated with recognition memory performance. However, we also observed some gamma-band synchronization before stimulus onset. Nonparametric randomization tests applied to the prestimulus period revealed that neither gamma-band coherence nor power was correlated with subsequent recognition memory performance during this period (supplemental Figs. 2, 3, 5, 6, available at www. jneurosci.org as supplemental material), suggesting that the observed level of prestimulus synchronization reflects hippocampal processing unrelated to the behavioral task. One possibility is that this prestimulus synchronization reflects arousal mechanisms. Timing was held constant throughout the experiment (1 s fixation period), so it would be possible for the monkeys to anticipate the onset of the stimulus. Alternatively, between the end of the previous stimulus presentation and the beginning of the next, the monkey may be engaged in retrieving previous stimuli, encoding new information, or some other uncontrolled process. Because some hippocampal function is likely during such processes, the presence of spike-field coherence during this interval is not wholly surprising. Functional imaging studies often use a separate task during baseline periods because the use of a simple "rest" period can potentially lead to high levels of hippocampal activation (Stark and Squire, 2001).

Although several studies have identified activity at the cellular level related to recognition memory in the cortex surrounding the hippocampus (Miller et al., 1991, 1993; Riches et al., 1991; Sobotka and Ringo, 1993; Suzuki et al., 1997), there is a notable lack of evidence for recognition memory signals in the hippocampus proper. One important difference between our task and those used in previous neurophysiological studies is the degree of training involved. The VPLT requires only simple fixation training. In contrast, the tasks used in previous neurophysiology studies require a long period of training (up to 7-10 months), during which monkeys gradually learn a match-to-sample rule. It is conceivable that, during this training period, monkeys acquire strategies for performing the task that do not rely on the hippocampus. Similarly, whereas the VPLT examines the monkey's innate preference for novelty, tasks in previous studies examined the monkey's ability to respond correctly to receive a food or juice reward. The reward component of these tasks may encourage the acquisition of strategies that recruit extrahippocampal structures. Consistent with this idea, the VPLT has been shown to be more sensitive than the delayed nonmatching-to-sample task to restricted lesions of the hippocampus (Pascalis and Bachevalier, 1999; Zola et al., 2000; Nemanic et al., 2004).
In summary, we have used spectral analysis to examine the role of precise spike timing in the hippocampus in memory formation. Our results are consistent with the idea that memory encoding in the medial temporal lobe relies on a combination of firing rate changes at the single-cell level and altered patterns of synchronization at the population level.

\section{References}

Baxter MG, Murray EA (2001a) Opposite relationship of hippocampal and rhinal cortex damage to delayed nonmatching-to-sample deficits in monkeys. Hippocampus 11:61-71.

Baxter MG, Murray EA (2001b) Effects of hippocampal lesions on delayed nonmatching-to-sample in monkeys: a reply to Zola and Squire (2001) Hippocampus 11:201-203.

Bi GQ, Poo MM (1998) Synaptic modifications in cultured hippocampal neurons: dependence on spike timing, synaptic strength, and postsynaptic cell type. J Neurosci 18:10464-10472.

Bichot NP, Rossi AF, Desimone R (2005) Parallel and serial neural mechanisms for visual search in macaque area V4. Science 308:529-534.

Bragin A, Jandó G, Nádasdy Z, Hetke J, Wise K, Buzsáki G (1995) Gamma $(40-100 \mathrm{~Hz})$ oscillation in the hippocampus of the behaving rat. J Neurosci 15:47-60.

Brown MW, Wilson FA, Riches IP (1987) Neuronal evidence that inferomedial temporal cortex is more important than hippocampus in certain processes underlying recognition memory. Brain Res 409:158-162.

Buschman TJ, Miller EK (2007) Top-down versus bottom-up control of attention in the prefrontal and posterior parietal cortices. Science 315:1860-1862.

Buzsáki G (2004) Large-scale recording of neuronal ensembles. Nat Neurosci 7:446-451.

Canolty RT, Edwards E, Dalal SS, Soltani M, Nagarajan SS, Kirsch HE, Berger MS, Barbaro NM, Knight RT (2006) High gamma power is phaselocked to theta oscillations in human neocortex. Science 313:1626-1628.

Chrobak JJ, Buzsáki G (1996) High-frequency oscillations in the output networks of the hippocampal-entorhinal axis of the freely behaving rat. J Neurosci 16:3056-3066.

Csicsvari J, Jamieson B, Wise KD, Buzsáki G (2003) Mechanisms of gamma oscillations in the hippocampus of the behaving rat. Neuron 37:311-322.

Edwards E, Soltani M, Deouell LY, Berger MS, Knight RT (2005) High gamma activity in response to deviant auditory stimuli recorded directly from human cortex. J Neurophysiol 94:4269-4280.

Fell J, Klaver P, Lehnertz K, Grunwald T, Schaller C, Elger CE, Fernández G (2001) Human memory formation is accompanied by rhinal-hippocampal coupling and decoupling. Nat Neurosci 4:1259-1264.

Fell J, Dietl T, Grunwald T, Kurthen M, Klaver P, Trautner P, Schaller C, Elger CE, Fernández G (2004) Neural bases of cognitive ERPs: more than phase reset. J Cogn Neurosci 16:1595-1604.

Fries P (2005) A mechanism for cognitive dynamics: neuronal communications through neuronal coherence. Trends Cogn Sci 9:474-480.

Fries P, Reynolds JH, Rorie AE, Desimone R (2001) Modulation of oscillatory neuronal synchronization by selective visual attention. Science 291:1560-1563.

Fries P, Womelsdorf T, Oostenveld R, Desimone R (2008) The effects of visual stimulation and selective visual attention on rhythmic neuronal synchronization in macaque area V4. J Neurosci 28:4823-4835.

Halgren E, Marinkovic K, Chauvel P (1998) Generators of the late cognitive potentials in auditory and visual oddball tasks. Electroencephalogr Clin Neurophysiol 106:156-164.

Hoogenboom N, Schoffelen J-M, Oostenveld R, Parkes LM, Fries P (2006) Localizing human visual gamma-band activity in frequency, time and space. Neuroimage 29:764-773.

Jarvis MR, Mitra PP (2001) Sampling properties of the spectrum and coherency of sequences of action potentials. Neural Comput 13:717-749.

Kilner JM, Baker SN, Salenius S, Hari R, Lemon RN (2000) Human cortical muscle coherence is directly related to specific motor parameters. J Neurosci 20:8838-8845.

Knight R (1996) Contribution of human hippocampal region to novelty detection. Nature 383:256-259.

Kok A (2001) On the utility of P3 amplitude as a measure of processing capacity. Psychophysiology 38:557-577.

Manns JR, Hopkins RO, Reed JM, Kitchener EG, Squire LR (2003) Recognition memory and the human hippocampus. Neuron 37:171-180. 
Maris E, Oostenveld R (2007) Nonparametric statistical testing of EEG- and MEG-data. J Neurosci Methods 164:177-190.

Markram H, Lübke J, Frotscher M, Sakmann B (1997) Regulation of synaptic efficacy by coincidence of postsynaptic APs and EPSPs. Science 275: 213-215.

McKee RD, Squire LR (1993) On the development of declarative memory. J Exp Psychol Learn Mem Cogn 19:397-404.

Miller EK, Li L, Desimone R (1991) A neural mechanism for working and recognition memory in inferior temporal cortex. Science 254:1377-1379.

Miller EK, Li L, Desimone R (1993) Activity of neurons in anterior inferior temporal cortex during a short-term memory task. J Neurosci 13:14601478.

Mishra J, Fellous JM, Sejnowski TJ (2006) Selective attention through phase relationship of excitatory and inhibitory input synchrony in a model cortical neuron. Neural Netw 19:1329-1346.

Mitra PP, Pesaran B (1999) Analysis of dynamic brain imaging data. Biophys J 76:691-708.

Montgomery SM, Buzsáki G (2007) Gamma oscillations dynamically couple hippocampal CA3 and CA1 regions during memory task performance. Proc Natl Acad Sci U S A 104:14495-14500.

Murray EA, Mishkin M (1998) Object recognition and location memory in monkeys with excitotoxic lesions of the amygdala and hippocampus. J Neurosci 18:6568-6582.

Nemanic S, Alvarado MC, Bachevalier J (2004) The hippocampal/parahippocampal regions and recognition memory: insights from visual paired comparison versus object-delayed nonmatching in monkeys. J Neurosci 24:2013-2026.

Nichols TE, Holmes AP (2002) Nonparametric permutation tests for functional neuroimaging: a primer with examples. Hum Brain Mapp 15:1-25.

Niessing J, Ebisch B, Schmidt KE, Niessing M, Singer W, Galuske RAW (2005) Hemodynamic signals correlate tightly with synchronized gamma oscillations. Science 309:948-951.

Pascalis O, Bachevalier J (1999) Neonatal aspiration lesions of the hippocampal formation impair visual recognition memory when assessed by paired-comparison task but not by delayed nonmatching-to-sample task. Hippocampus 9:609-616.

Pesaran B, Pezaris JS, Sahani M, Mitra PP, Andersen RA (2002) Temporal structure in neuronal activity during working memory in macaque parietal cortex. Nat Neurosci 5:805-811.

Polich J (2007) Updating P300: an integrative theory of P3a and P3b. Clin Neurophysiol 118:2128-2148.

Riches IP, Wilson FA, Brown MW (1991) The effects of visual stimulation and memory on neurons of the hippocampal formation and the neighboring parahippocampal gyrus and inferior temporal cortex of the primate. J Neurosci 11:1763-1779.

Robbe D, Montgomery SM, Thome A, Rueda-Orozco PE, McNaughton BL, Buzsáki G (2006) Cannabinoids reveal importance of spike timing coordination in hippocampal function. Nat Neurosci 9:1526-1533.

Rolls ET, Miyashita Y, Cahusac PM, Kesner RP, Niki H, Feigenbaum JD, Bach L (1989) Hippocampal neurons in the monkey with activity related to the place in which a stimulus is shown. J Neurosci 9:1835-1845.

Rolls ET, Cahusac PM, Feigenbaum JD, Miyashita Y (1993) Responses of single neurons in the hippocampus of the macaque related to recognition memory. Exp Brain Res 93:299-306.

Rosenberg JR, Amjad AM, Breeze P, Brillinger DR, Halliday DM (1989) The Fourier approach to the identification of functional coupling between neuronal spike trains. Prog Biophys Mol Biol 53:1-31.
Rutishauser U, Mamelak AN, Schuman EM (2006) Single-trial learning of novel stimuli by individual neurons of the human hippocampusamygdala complex. Neuron 49:805-813.

Salinas E, Sejnowski TJ (2000) Impact of correlated synaptic input on output firing rate and variability in simple neuronal models. J Neurosci 20:6193-6209.

Sederberg PB, Schulze-Bonhage A, Madsen JR, Bromfield EB, McCarthy DC, Brandt A, Tully MS, Kahana MJ (2007a) Hippocampal and neocortical gamma oscillations predict memory formation in humans. Cereb Cortex 17:1190-1196.

Sederberg PB, Schulze-Bonhage A, Madsen JR, Bromfield EB, Litt B, Brandt A, Kahana MJ (2007b) Gamma oscillations distinguish true from false memories. Psychol Sci 18:927-932.

Sirota A, Montgomery S, Fujisawa S, Isomura Y, Zugaro M, Buzsáki G (2008) Entrainment of neocortical neurons and gamma oscillations by the hippocampal theta rhythm. Neuron 60:683-697.

Sobotka S, Ringo JL (1993) Investigation of long-term recognition and association memory in unit responses from inferotemporal cortex. Exp Brain Res 96:28-38.

Soltani M, Knight RT (2000) Neural origins of the P300. Crit Rev Neurobiol 14:199-224.

Stark CE, Squire LR (2001) When zero is not zero: the problem of ambiguous baseline conditions in fMRI. Proc Natl Acad Sci U S A 98:1276012766.

Suzuki WA, Miller EK, Desimone R (1997) Object and place memory in the macaque entorhinal cortex. J Neurophysiol 78:1062-1081.

Taylor K, Mandon S, Freiwald WA, Kreiter AK (2005) Coherent oscillatory activity in monkey area V4 predicts successful allocation of attention. Cereb Cortex 15:1424-1437.

Wilson FA, Goldman-Rakic PS (1994) Viewing preferences of rhesus monkeys related to memory for complex pictures, colours and faces. Behav Brain Res 60:79-89.

Womelsdorf T, Fries P, Mitra PP, Desimone R (2006) Gamma-band synchronization in visual cortex predicts speed of change detection. Nature 439:733-736.

Womelsdorf T, Schoffelen J-M, Oostenveld R, Singer W, Desimone R, Engel AK, Fries P (2007) Modulation of neuronal interactions through neuronal synchronization. Science 316:1609-1612.

Wyart V, Tallon-Baudry C (2008) Neural dissociation between visual awareness and spatial attention. J Neurosci 28:2667-2679.

Wyart V, Tallon-Baudry C (2009) How ongoing fluctuations in human visual cortex predict perceptual awareness: baseline shift versus decision bias. J Neurosci 29:8715-8725.

Xiang JZ, Brown MW (1998) Differential neuronal encoding of novelty, familiarity and recency in regions of the anterior temporal lobe. Neuropharmacology 37:657-676.

Zeitler M, Fries P, Gielen S (2006) Assessing neuronal coherence with single-unit, multi-unit, and local field potentials. Neural Comput 18:2256-2281.

Zola SM, Squire LR (2001) Relationship between magnitude of damage to the hippocampus and impaired recognition memory in monkeys. Hippocampus 11:92-98.

Zola SM, Squire LR, Teng E, Stefanacci L, Buffalo EA, Clark RE (2000) Impaired recognition memory in monkeys after damage limited to the hippocampal region. J Neurosci 20:451-463. 